Discrete Comput Geom 29:603-623 (2003)

DOI: $10.1007 / \mathrm{s} 00454-003-0778-7$

\title{
The Heighway Dragon Revisited
}

\author{
Sze-Man Ngai ${ }^{1}$ and Nhu Nguyen ${ }^{2}$ \\ ${ }^{1}$ Department of Mathematics and Computer Science, Georgia Southern University, \\ Statesboro, GA 30460-8093, USA \\ ngai@gsu.cs.gasou.edu \\ ${ }^{2}$ Department of Mathematics, New Mexico State University, \\ Las Cruces, NM 88003-8001, USA \\ nnguyen@nmsu.edu
}

\begin{abstract}
We prove that the Heighway dragon is a countable union of closed geometrically similar disk-like planar sets which intersect each other in a linear order: any two of them intersect at no more than one cut point and for any three disks there exist at least two with an empty intersection. Consequently, the interior of the Heighway dragon is a countable union of disjoint open disk-like planar sets. We determine all the cut points of the dragon and show that each disk-like subset between two cut points is a graph self-similar set defined by a graph-directed iterated function system consisting of four seed sets. Our results describe a fairly complete picture of the topological and geometric structure of the Heighway dragon.
\end{abstract}

\section{Introduction}

The $n$th Heighway dragon curve $\mathcal{P}_{n}$ is a planar curve obtained by an $n$-fold iteration of the unit vertical line segment $\mathcal{P}_{0}:=\{(0, y): 0 \leq y \leq 1\} \subset \mathbb{R}^{2}$. The iteration is defined as follows (see Fig. 1(a)): $\mathcal{P}_{1}$ is obtained by replacing $\mathcal{P}_{0}$ with two line segments of equal length $1 / \sqrt{2}$ joined at a right angle, with $\mathcal{P}_{1}$ on the left of $\mathcal{P}_{0}$, and with the same two ends as $\mathcal{P}_{0} . \mathcal{P}_{n}$ is obtained by iterating $\mathcal{P}_{n-1}$ left and right alternately, starting with left, counting from the lower endpoint $(0,0)$.

* The first author was supported in part by the National Science Foundation, Grant DMS-96-32032, an HKRGC grant, a Faculty Research Grant from Georgia Southern University, and a Foundation Grant from the Department of Mathematics and Computer Science of Georgia Southern University. The second author was supported by the Minigrant RC01-014 of New Mexico State University; this author has previously published as Nguyen To Nhu. 


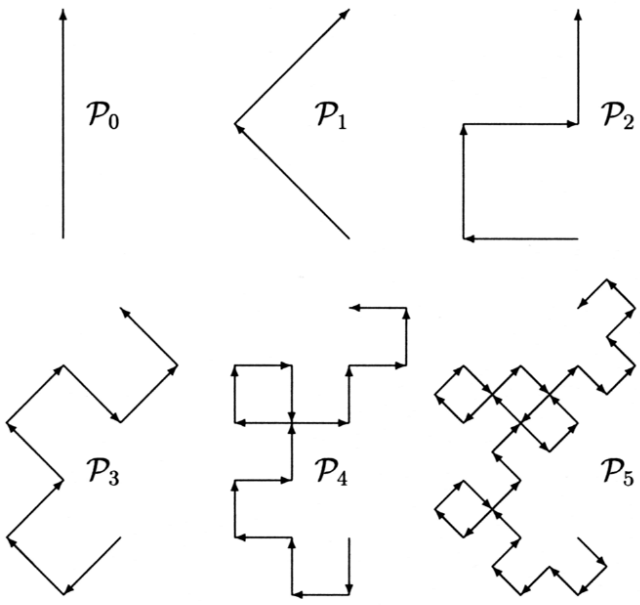

(a)

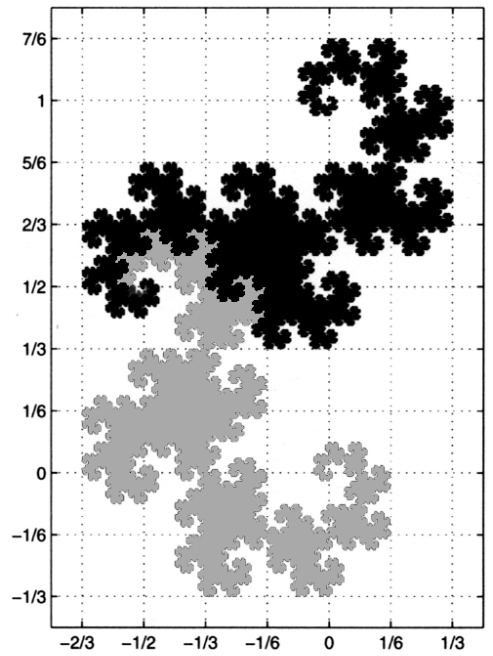

(b)

Fig. 1. (a) The $n$th Heighway dragon curves $\mathcal{P}_{n}$ for $n=0,1, \ldots, 5$. (b) The Heighway dragon as a 2-reptile.

$\left\{\mathcal{P}_{n}\right\}$ is a Cauchy sequence of compact sets in $\mathbb{R}^{2}$ in the Hausdorff metric (see, e.g., $[\mathrm{F}])$. It is well known that the space of nonempty compact sets in $\mathbb{R}^{d}$ equipped with the Hausdorff metric is complete. The limit (in the Hausdorff metric) of $\left\{\mathcal{P}_{n}\right\}$ as $n \rightarrow \infty$, denoted by $D$ throughout this paper, is called the Heighway dragon (see Fig. 1(b)).

The Heighway dragon $D$ was discovered by physicist John E. Heighway and was later analyzed by Harter, Heighway, and Banks in the 1960s (see [G]). In was studied rigorously by Davis and Knuth $[\mathrm{DK}]$ in connection with number systems and paperfolding. They proved that copies of $D$ tile the plane; therefore $D$ has a nonempty interior. In fact, the well-known twin dragon is a union of two Heighway dragons (see [E]); this fact is used in Section 4 of the paper. The twin dragon is a topological disk (see, e.g., [BW]).

The Heighway dragon $D$ is an interesting fractal set (see [M] and [E]). In fact it is the self-similar set invariant under the iterated function system (IFS) (see $[\mathrm{H}]$ and $[\mathrm{F}]$ ) $\varphi_{1}, \varphi_{2}: \mathbb{R}^{2} \rightarrow \mathbb{R}^{2}$ defined as

$$
\varphi_{1}(x)=\frac{1}{\sqrt{2}} R\left(\frac{\pi}{4}\right) x, \quad \varphi_{2}(x)=\frac{1}{\sqrt{2}} R\left(\frac{3 \pi}{4}\right) x+\left[\begin{array}{l}
0 \\
1
\end{array}\right],
$$

where $R(\theta): \mathbb{R}^{2} \rightarrow \mathbb{R}^{2}$ is the counterclockwise rotation by $\theta$. Thus, $D$ satisfies the self-similar identity

$$
D=\varphi_{1}(D) \cup \varphi_{2}(D) .
$$

It is known (see [E]) that the Hausdorff dimension of the boundary of $D$ is equal to $2 \log \lambda / \log 2=1.5236270862 \ldots$, where $\lambda$ is the largest real zero of $\lambda^{3}-\lambda^{2}-2$.

More recently the Heighway dragon arises naturally in the study of self-similar tilings (see [B], [BP], [SW], and [NSVW]). It is a well-known 2-reptile. Benedek and Panzone 
[BP] showed that its Lebesgue measure is equal to $\frac{1}{2}$ and determined its convex hull. Topologically, it is known that all 2-repsets in $\mathbb{R}^{d}$ are connected and therefore $D$ is connected (see [KL] and [NSVW]); moreover, it is the closure of its interior.

Much work has been carried out regarding the topological structure of self-similar tiles and more generally self-affine tiles, especially in determining conditions under which a self-affine tile is a topological disk (see [BG], [Ge1], [Ge2], [BW], and [LRT]). However, few results on the structure of non-disk-like tiles are available, and this partly motivates our work. Out of the six rational 2-reptiles (assuming that no reflections are involved) found in [NSVW], the Heighway dragon and the Lévy dragon are the only two that are not topological disks. Recently, the structure of the Lévy dragon was explored by Bailey et al. [BKS].

Despite the above investigations, there are still interesting unanswered questions concerning the topological structure of the Heighway dragon. It is convincing from the picture that its interior is disconnected and consists of countably infinite simply connected components. The main purpose of this paper is to prove these observations, and more, rigorously.

Theorem 1.1 (Main Theorem). The Heighway dragon D is the closure of a countable union of geometrically similar closed disk-like planar sets which intersect each other in a linear order: any two of them intersect at no more than one point and for any three disks there exist at least two with an empty intersection.

Corollary 1.2. The interior of $D$ is a countable union of disjoint open disk-like planar sets.

A point $c$ in a connected set $E \subseteq \mathbb{R}^{2}$ is a cut point of $E$ if $E \backslash\{c\}$ is disconnected. We say that two cut points $a, b \in D$ are consecutive if they divide $D$ into three mutually disjoint connected parts in such a way that the part containing both $a$ and $b$ in its closure does not have any cut points. We study the structure of $D$ by analyzing the central piece $C$ of $D$ (see Section 3), which is the largest connected subset of $D$ without cut points, together with the convex hull $H$ of $C$. We show in Section 4 that $C$ is the attractor of a graph-directed IFS with four seed sets: a twin dragon, a contracted copy of $C$ itself by a linear factor $\frac{1}{2}$, and two contracted copies of $C$ by a linear factor $\frac{1}{4}$. We will also show that $H$ is a 10 -sided polygon and determine all its vertices $\left\{e_{i}: i=1, \ldots, 10\right\}$ (Proposition 3.4), where $e_{4}=c_{0}:=\left(-\frac{1}{3}, \frac{1}{3}\right)$ and $e_{8}=c_{1}:=\left(0, \frac{2}{3}\right)$ are cut points of $D$ (Proposition 3.2).

Throughout this paper we let $C_{0}:=C, C_{-\infty}:=\{(0,0)\}, C_{\infty}:=\{(0,1)\}, H_{0}:=H$, and for all $n \geq 1$ define

$$
\begin{aligned}
C_{-n}:=\varphi_{1}^{n}(C), & C_{n}:=\varphi_{2} \circ \varphi_{1}^{n}(C), \\
H_{-n}:=\varphi_{1}^{n}(H), & H_{n}:=\varphi_{2} \circ \varphi_{1}^{n}(H), \\
c_{-n}:=\varphi_{1}^{n}\left(c_{0}\right), & c_{n}:=\varphi_{2} \circ \varphi_{1}^{n-1}\left(c_{0}\right) .
\end{aligned}
$$

Note by similarity that for all $n \in \mathbb{Z}, H_{n}$ is the convex hull of $C_{n}$. Theorem 1.1 will be proved by the following two theorems. 
Theorem 1.3. Let $C_{n}, H_{n}$, and $c_{n}$ be defined as above. The following statements hold:

(a) $D=\bigcup_{n \in \mathbb{Z} \cup\{-\infty, \infty\}} C_{n}$.

(b) The set of all cut points in $D$ is the countable set $\left\{c_{n}\right\}_{n=-\infty}^{\infty}$.

(c) For each $n \in \mathbb{Z}, H_{n}$ (resp. $C_{n}$ ) has nonempty intersection only with $H_{n-1}$ (resp. $\left.C_{n-1}\right)$ and $H_{n+1}$ (resp. $\left.C_{n+1}\right)$ at $c_{n}$ and $c_{n+1}$, respectively. Moreover, the four boundary sides of $H_{n}$ and $H_{n-1}$ intersecting $c_{n}$ align to form two line segments.

(d) The precise Cartesian coordinates of $c_{n}$ are

$$
\begin{aligned}
c_{n}= & \left(\left(\frac{1}{3}\right) 2^{-(n-1) / 2} \cos [(n-3) \pi / 4], 1+\left(\frac{1}{3}\right) 2^{-(n-1) / 2} \sin [(n-3) \pi / 4]\right), \\
& n \geq 1, \\
c_{-n}= & \left(\left(\frac{1}{3}\right) 2^{-(n-1) / 2} \cos [(n+3) \pi / 4],\left(\frac{1}{3}\right) 2^{-(n-1) / 2} \sin [(n+3) \pi / 4]\right), \\
& n \geq 0 .
\end{aligned}
$$

Moreover, $c_{n}$ and $c_{n+1}, n \in \mathbb{Z}$, are "consecutive" in the sense of the above definition.

Theorem 1.4. The region between any two consecutive cut points in $D$ is a closed topological disk.

This paper is organized as follows. Section 2 establishes some basic properties of the dragon curves $\mathcal{P}_{n}$. We prove, among other results, that two nearest vertices of $\mathcal{P}_{n}$ must be connected by a curve in $\mathcal{P}_{n}$ consisting of at most three edges of $\mathcal{P}_{n}$ (Proposition 2.2). Section 3 studies the central piece $C$. We show that it is the attractor of a graph-directed IFS and find its convex hull. In Section 4 we show that the interior of $C$ is an open topological disk. Section 5 is devoted to the proof of the main results.

\section{Heighway Dragon Curves and Their Iterations}

The line segments in $\mathcal{P}_{n}$ are called the edges of $\mathcal{P}_{n}$, and the endpoints of the edges are called the vertices of $\mathcal{P}_{n}$. Let $E\left(\mathcal{P}_{n}\right)$ and $V\left(\mathcal{P}_{n}\right)$ denote respectively the set of edges and the set of vertices of $\mathcal{P}_{n}$. Each edge of $\mathcal{P}_{n}$ is of length $2^{-n / 2}$. Any two edges in $\mathcal{P}_{n}$ are either parallel or perpendicular. Also, $V\left(\mathcal{P}_{n}\right) \subset V\left(\mathcal{P}_{n+1}\right)$ for all $n \geq 0$.

For each $n \geq 0, \mathcal{P}_{n}$ lies on a uniquely determined $2^{-n / 2}$-mesh of $\mathbb{R}^{2}$ consisting of parallel and perpendicular lines. We denote this mesh by $\mathcal{M}_{n}$. The definitions of edges and vertices generalize naturally to $\mathcal{M}_{n}$; we use the analogous notation $E\left(\mathcal{M}_{n}\right)$ and $V\left(\mathcal{M}_{n}\right)$.

The initial vertex of $\mathcal{P}_{n}$ is the lower endpoint $(0,0)$ and the terminal vertex is the upper endpoint $(0,1)$. The edges containing the initial and terminal vertices are called the initial edge and terminal edge, respectively. The directed graph from the initial vertex to the terminal vertex obtained by the iteration process is called the principal path of $\mathcal{P}_{n}$. We denote an edge in $E\left(\mathcal{P}_{n}\right)$ with endpoints $a, b \in V\left(\mathcal{P}_{n}\right)$ by $[a, b]$ or $[a \rightarrow b]$ if we need to indicate the direction of the principal path.

Observe that the principal path visits all edges of $\mathcal{P}_{n}$ without crossing any edge or visiting an edge twice (see Proposition 1.5.7 of [E]). Therefore it must turn left or right 
at every corner. It follows that $\mathcal{P}_{n}$ consists of $2^{n}$ edges, divided into two groups each consisting of $2^{n-1}$ edges. Members in the same group are parallel and those in different groups are perpendicular. Since the $n$th iteration starts by iterating the initial edge of $\mathcal{P}_{n}$ to the left, it must end by iterating the terminal edge to the right. All edges parallel to the initial edge iterate to the left, and all edges perpendicular to the initial edge iterate to the right. We denote these two groups of edges by $E_{\ell}\left(\mathcal{P}_{n}\right)$ and $E_{\mathrm{r}}\left(\mathcal{P}_{n}\right)$, respectively.

For each subset $E \subseteq \mathbb{R}^{d}$, we denote by $E^{\circ}, \bar{E}, \partial E$, and $\operatorname{diam}(E)$ the interior, closure, boundary, and diameter of $E$, respectively. Besides the Hausdorff metric the following distance $d(E, F)$ between two sets $E, F \subseteq \mathbb{R}^{d}$ will also be used frequently throughout the paper:

$$
d(E, F):=\inf \{\|x-y\|: x \in E, y \in F\} .
$$

\section{Observation 2.1.}

(a) A vertex in $V\left(\mathcal{P}_{n}\right)$ cannot intersect exactly three edges or exactly two collinear edges in $E\left(\mathcal{P}_{n}\right)$. If it intersects four edges in $E\left(\mathcal{P}_{n}\right)$, then two collinear edges must point toward and the other two must point away from the vertex.

(b) For any $a, b \in V\left(\mathcal{P}_{n}\right)$,

$$
\|a-b\|=2^{-n / 2} \sqrt{p^{2}+q^{2}}
$$

where $p$ and $q$ are non-negative integers.

(c) If $[a, b] \in E\left(\mathcal{P}_{n}\right), n \geq 1$, then one of the ends a or $b$ must be a vertex of $\mathcal{P}_{n-1}$ and the other must be a vertex in $V\left(\mathcal{P}_{n}\right) \backslash V\left(\mathcal{P}_{n-1}\right)$.

Proof. (a) Observe, by induction, that the initial and terminal vertices lie only on the initial and terminal edges, respectively. Consequently, if a vertex in $\mathcal{P}_{n}$ intersects exactly three edges in $\mathcal{P}_{n}$, then one of these edges would be traversed by the principal path more than once, which is impossible. Since the principal path must turn left or right at every corner, a vertex of $\mathcal{P}_{n}$ cannot intersect exactly two collinear edges.

For the last assertion we first observe that if more than two edges point toward or away from a vertex, then one of them would have to be an initial or terminal edge, which is impossible. Now suppose a collinear pair of the four edges belong to, say, $E_{\ell}\left(\mathcal{P}_{n}\right)$, and have the same direction. (The other pair must belong to $E_{\mathrm{r}}\left(\mathcal{P}_{n}\right)$.) Then upon a further iteration, one of the edges in $\mathcal{P}_{n+1}$ would be traversed more than once, which is again impossible.

(b) Since all edges of $\mathcal{P}_{n}$ lie on a mesh of squares of side length $2^{-n / 2},(2.2)$ follows from the Pythagorean Theorem.

(c) Assume without loss of generality that $[a, b]=[a \rightarrow b]$. Since $[a, b]$ must come from iterating some $[c, d]=[c \rightarrow d] \in E\left(\mathcal{P}_{n-1}\right)$, either $a=c$ or $b=d$. The assertion follows.

We call a connected curve $\mathcal{P}_{n}$ consisting of finitely many edges in $\mathcal{P}_{n}$ a subcurve of $\mathcal{P}_{n}$. By a small square in $\mathcal{P}_{n}$ we mean a square in $\mathcal{P}_{n}$ formed by exactly four edges of $\mathcal{P}_{n}$. Subcurves and small squares of $\mathcal{M}_{n}$ are defined similarly. We denote the vertices and edges in a subcurve $\mathcal{C}$ by $V(\mathcal{C})$ and $E(\mathcal{C})$, respectively. 
(a)

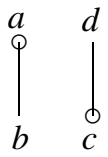

(b)

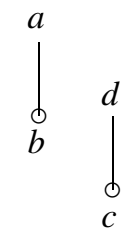

Fig. 2. The two possible configurations in the proof of Proposition 2.2. Circles represent vertices in $\mathcal{P}_{n-1}$.

Proposition 2.2. Let $[a, b],[c, d] \in E\left(\mathcal{P}_{n}\right)$. If $d([a, b],[c, d])=2^{-n / 2}$, then there exists a subcurve of $\mathcal{P}_{n}$ consisting of at most three edges connecting $[a, b]$ and $[c, d]$. Moreover, if $[a, b]$ and $[c, d]$ are parallel and noncollinear, then they are connected by exactly one edge of $\mathcal{P}_{n}$.

Proof. By induction it is easy to see that the proposition holds if one of $[a, b]$ or $[c, d]$ is an initial or terminal edge. So we assume that this is not the case. We prove the proposition by induction on $n$. It holds obviously for $n=0,1, \ldots, 5$ and even larger $n$ (see Fig. 1(a)). Assume that it holds for $n-1$. By replacing the edges if necessary, it suffices to consider the case when $[a, b]$ and $[c, d]$ are parallel and noncollinear.

Without loss of generality we may assume that $[a, b]$ and $[c, d]$ are vertical line segments with $a, d$ on the top and $b, c$ on the bottom. Then either $[a, b] \perp[b, c]$ or $[a, b] \perp[b, d]$. By Observation 2.1(c) we assume that $a \in V\left(\mathcal{P}_{n-1}\right)$.

Case 1: $[a, b] \perp[b, c]$; see Fig. 2(a). Since two vertices of $P_{n-1}$ must be at least $2^{-(n-1) / 2}$ apart, it follows that $c \in V\left(\mathcal{P}_{n-1}\right)$, and $\|a-c\|=2^{-(n-1) / 2}$. Thus by induction assumption either $[a, c]$ is an edge of $\mathcal{P}_{n-1}$ or $a$ and $c$ can be connected by three edges of $\mathcal{P}_{n-1}$. In the former case the two edges in $\mathcal{P}_{n}$ arising from iterating $[a, c]$ would connect either $a$ to $d$ or $b$ to $c$. The same conclusion holds in the latter case after iterating the three edges and using Observation 2.1(a) if necessary.

Case 2: $[a, b] \perp[b, d]$; see Fig. 2(b). By Observation 2.1(c) either $c$ or $d$ is a vertex of $\mathcal{P}_{n-1}$. The case $d \in V\left(\mathcal{P}_{n-1}\right)$ is similar to Case 1 . If $c \in V\left(\mathcal{P}_{n-1}\right)$, then by Observation 2.1(b),

$$
2^{-n / 2} \sqrt{5}=\|a-c\|=2^{-(n-1) / 2} \sqrt{p^{2}+q^{2}}
$$

for some non-negative integers $p$ and $q$, which is clearly impossible.

We now turn to iterations of the $\mathcal{P}_{n}$. If $\mathcal{C}$ is a subcurve of $\mathcal{P}_{n}$, let $\sigma^{k}(\mathcal{C})$ denote the part of $\mathcal{P}_{n+k}$ obtained by iterating $\mathcal{C}$ for $k$ times, with

$$
\sigma^{0}(\mathcal{C}):=\mathcal{C}, \quad \sigma^{1}(\mathcal{C}):=\sigma(\mathcal{C}), \quad \text { and } \quad \sigma^{\infty}(\mathcal{C}):=\lim _{k \rightarrow \infty} \sigma^{k}(\mathcal{C})
$$


where the limit is taken in the Hausdorff metric. The following lemma will be used frequently:

Lemma 2.3. Let $\mathcal{S}, \mathcal{T}$ be subcurves of $\mathcal{P}_{n}$ and let $E \subseteq \mathbb{R}^{2}$ be nonempty. We have:

(a) $d\left(\sigma^{k}(\mathcal{S}), E\right) \geq d(\mathcal{S}, E)-2^{-n / 2+1} / 3$ for every $k \geq 0$.

(b) $d\left(\sigma^{k}(\mathcal{S}), \sigma^{k}(\mathcal{T})\right) \geq d(\mathcal{S}, \mathcal{T})-2^{-n / 2+2} / 3$ for every $k \geq 0$.

(c) Let $\mathcal{S} \in E_{\ell}\left(\mathcal{P}_{n}\right)$. Then the part of the Heighway dragon obtained by iterating $\mathcal{S}$ is bounded by the rectangle determined by the four straight lines: two are parallel to $\mathcal{S}$ and of distances $2^{-n / 2+1} / 3$ and $2^{-n / 2} / 3$ from the left and right of $\mathcal{S}$, respectively; the other two are perpendicular to $\mathcal{S}$ and of distances $2^{-n / 2} / 3$ and $2^{-n / 2-1} / 3$ from the bottom and top of $\mathcal{S}$, respectively. If $\mathcal{S} \in E_{\mathrm{r}}\left(\mathcal{P}_{n}\right)$, then the bounding rectangle of $\mathcal{S}$ can be determined as above by first reversing the direction of $\mathcal{S}$.

Proof. We prove (a). Parts (b) and (c) can be proved similarly. Observe that after the first four iterations we have (see Fig. 1(a))

$$
d\left(\sigma^{k}(\mathcal{S}), E\right) \geq d(\mathcal{S}, E)-2^{-n / 2-1} \quad \text { for } \quad k=1,2,3,4 .
$$

In general, for every $k \leq 4 m+4$ we have

$$
d\left(\sigma^{k}(\mathcal{S}), E\right) \geq d(\mathcal{S}, E)-\left(2^{-n / 2-1}+2^{-n / 2-3}+\cdots+2^{-n / 2-2 m-1}\right) .
$$

Consequently for every $k \geq 0$,

$$
d\left(\sigma^{k}(\mathcal{S}), E\right) \geq d(\mathcal{S}, E)-\sum_{m=0}^{\infty} 2^{-n / 2-2 m-1}=d(\mathcal{S}, E)-\frac{2^{-n / 2+1}}{3} .
$$

This proves (a) and the lemma is proved.

Proposition 2.4. Let $\mathcal{S}$ and $\mathcal{T}$ be two subcurves of $\mathcal{P}_{n}$ such that $\sigma^{\infty}(\mathcal{S}) \cap \sigma^{\infty}(\mathcal{T}) \neq \emptyset$. Then for every integer $k \geq 0$ there exists a subcurve of $\mathcal{P}_{n+k}$ consisting of at most three edges connecting $\sigma^{k}(\mathcal{S})$ with $\sigma^{k}(\mathcal{T})$.

Proof. Since any two edges of $\mathcal{P}_{n}$ are either parallel or perpendicular, the distance between $\sigma^{k}(\mathcal{S})$ and $\sigma^{k}(\mathcal{T})$ is actually the distance between some two vertices of $\mathcal{P}_{n+k}$. Therefore from Observation 2.1(b) we get

$$
d\left(\sigma^{k}(\mathcal{S}), \sigma^{k}(\mathcal{T})\right)=2^{-(n+k) / 2} \sqrt{p^{2}+q^{2}} \quad \text { for some integers } p, q .
$$

Since $\sigma^{\infty}(\mathcal{S}) \cap \sigma^{\infty}(\mathcal{T}) \neq \emptyset$, from Lemma 2.3(b) we get

$$
d\left(\sigma^{k}(\mathcal{S}), \sigma^{k}(\mathcal{T})\right) \leq \frac{2^{-(n+k) / 2+2}}{3} \quad \text { for all } \quad k \geq 0 .
$$

It follows that $\sqrt{p^{2}+q^{2}} \leq \frac{4}{3}$. Therefore $p^{2}+q^{2}$ equals 0 or 1 . In the former case $\sigma^{k}(\mathcal{S}) \cap \sigma^{k}(\mathcal{T}) \neq \emptyset$. In the latter case the assertion follows from Proposition 2.2. 
For a simple closed curve $\gamma$ in $\mathbb{R}^{2}$, we let $\Omega(\gamma)$ denote the closed topological disk enclosed by $\gamma$ (Jordan Curve Theorem). A simple closed curve $\mathcal{C}$ in $\mathcal{P}_{n}$ (or $\mathcal{M}_{n}$ ) will be called a circle in $\mathcal{P}_{n}$ (or $\mathcal{M}_{n}$, respectively). Let $\mathcal{C}$ be a circle in $\mathcal{M}_{n}$ and let $\mathcal{L}$ be a subcurve of $\mathcal{P}_{n}$ or $\mathcal{M}_{n} . \mathcal{L}$ is said to be inside $\mathcal{C}$ if it is enclosed by $\mathcal{C}$ and intersects $\mathcal{C}$ at no more than a finite number of points. $\mathcal{L}$ is strictly inside $\mathcal{C}$ if it is inside $\mathcal{C}$ and has an empty intersection with $\mathcal{C}$.

Lemma 2.5. Let $\mathcal{C}, \tilde{\mathcal{C}}$ be circles in $\mathcal{P}_{n}$ with $\tilde{\mathcal{C}}$ lying strictly inside $\mathcal{C}$. Assume that each edge of $\mathcal{M}_{n}$ inside $\mathcal{C}$ is an edge of $\mathcal{P}_{n}$. Then $\Omega(\tilde{\mathcal{C}}) \subseteq D^{\circ}$.

Proof. We first note that $d(\tilde{\mathcal{C}}, \mathcal{C}) \geq 2^{-n / 2}$ and thus by Lemma 2.3(a),

$$
d\left(\sigma^{k}(\mathcal{C}), \tilde{\mathcal{C}}\right) \geq \frac{2^{-n / 2}}{3} \quad \text { for every } \quad k \geq 0 .
$$

Hence, for any $k \geq 0, \Omega(\tilde{\mathcal{C}})$ lies in the interior of the region bounded by some circle $\mathcal{C}_{n+k}$ in $\mathcal{P}_{n+k}$ obtained by iterating $\mathcal{C}$, with $d\left(\tilde{\mathcal{C}}, \mathcal{C}_{n+k}\right) \geq 2^{-n / 2} / 3$. Next, since each edge in $\mathcal{M}_{n}$ enclosed by $\mathcal{C}$ belongs to $\mathcal{P}_{n}$ by assumption, it is easy to see that the analog holds for $\mathcal{C}_{n+k}$. In particular, each point in $\Omega(\tilde{\mathcal{C}})$ is of distance $\leq 2^{-(n+k) / 2}$ from the vertices $V\left(\mathcal{P}_{n+k}\right) \subseteq D$. The assertion now follows by letting $k \rightarrow \infty$.

We strengthen Lemma 2.5 in Section 4 (see Lemma 4.6).

\section{The Central Piece of the Heighway Dragon}

We begin by showing that $c_{0}=\left(-\frac{1}{3}, \frac{1}{3}\right)$ is a cut point of $D$. Denote by $R$ the filled rectangle bounding $D$ (Lemma 2.3(c)) and denote the intersections of $R$ with the lines passing through $c_{0}$ and parallel to the coordinate axes by

$$
f=\left(-\frac{2}{3}, \frac{1}{3}\right), \quad g=\left(\frac{1}{3}, \frac{1}{3}\right), \quad h=\left(-\frac{1}{3},-\frac{1}{3}\right), \quad k=\left(-\frac{1}{3}, \frac{7}{6}\right)
$$

(see Fig. 3(a)). For any $x, y \in \mathbb{R}^{2}$, let $[x, y]$ be the line segment connecting $x$ and $y$, and let $(x, y]:=[x, y] \backslash\{x\}$. Define $[x, y)$ and $(x, y)$ similarly.

Proposition 3.1. Let $[a, b]$ and $[d, e]$ be the edges of $\mathcal{P}_{4}$ shown in Fig. 3(a) and let $\mathcal{Q}$ denote the small square in Fig. 3(a) having $d$ as one of its vertices. Then:

(a) For any $k \leq \infty, \sigma^{k}([a, b])$ cannot hit the line segment $\left(c_{0}, g\right]$ from below, and $\sigma^{k}([d, e])$ cannot hit $\left[f, c_{0}\right)$ from above.

(b) For every $k \geq 0, d\left(\sigma^{k}(\mathcal{Q}),[f, g]\right) \geq \frac{1}{12}>0$.

Proof. (a) We prove the assertion for $[a, b]$. The proof for $[d, e]$ is similar. Observe that $[a, b] \in E_{\mathrm{r}}\left(\mathcal{P}_{4}\right)$. After four iterations we get a scaled copy of $[a, b]$, which belongs to $E_{\mathrm{r}}\left(\mathcal{P}_{8}\right)$. In fact, all such scaled copies of $[a, b]$ belong to $E_{\mathrm{r}}\left(\mathcal{P}_{4 m}\right)$.

For simplicity let $[a, b]$ denote a scaled copy of $[a, b]$ in $\mathcal{P}_{4 m}$. Denoting $\sigma([a, b])=$ $[a \rightarrow p \rightarrow b]$ we observe that

$$
d\left(\sigma^{k}([p, b]),\left[c_{0}, g\right]\right)=\left(\frac{1}{3}\right) 2^{-2 m} \quad \text { for } \quad k=0,1,2,3 .
$$



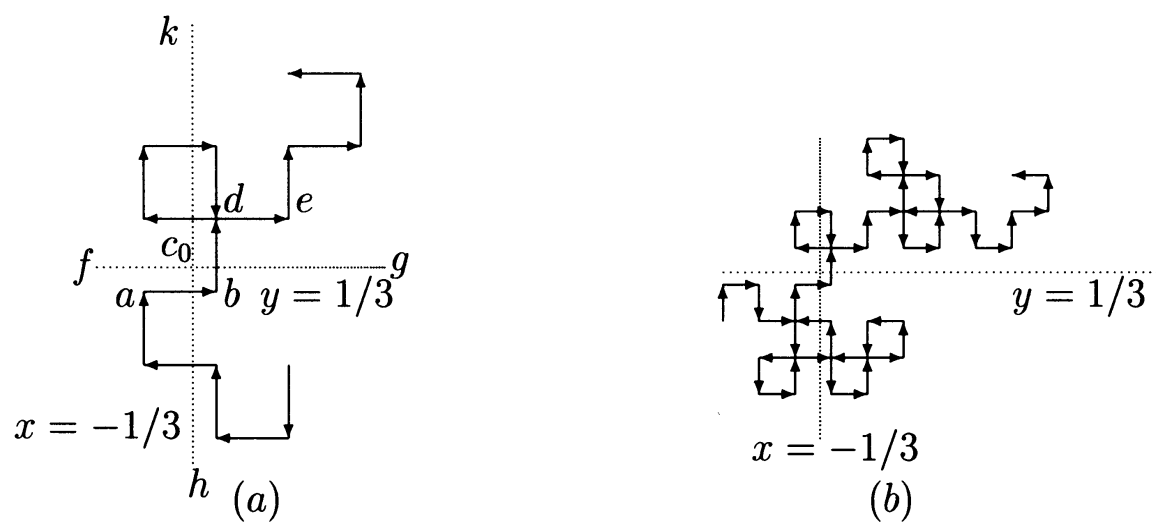

(b)

Fig. 3. (a) The curve $\mathcal{P}_{4}$. The intersection of the dotted lines is the point $c_{0}=\left(-\frac{1}{3}, \frac{1}{3}\right)$. (b) The part of $\mathcal{P}_{8}$ near the point $c_{0}$ (magnified). Only those edges generated by $[a, b],[b, d]$, and $[d, e]$ are shown.

Applying Lemma 2.3(a) with $n=4 m+4$ we get

$$
d\left(\sigma^{k}([p, b]),\left[c_{0}, g\right]\right) \geq\left(\frac{1}{3}\right) 2^{-2 m}-\left(\frac{1}{3}\right) 2^{-2 m-1}=\left(\frac{1}{3}\right) 2^{-2 m-1}>0
$$

for $k \leq \infty$. Consequently, $\sigma^{k}([p, b]) \cap\left[c_{0}, g\right]=\emptyset$ for $k \leq \infty$.

It remains to show that $\sigma^{k}([a, p]) \cap\left(c_{0}, g\right]=\emptyset$ for $k \leq \infty$. Observe that $\sigma([a, p])=$ $\left[a \rightarrow p^{\prime} \rightarrow p\right]$ consists of two edges $\left[a, p^{\prime}\right]$ and $\left[p^{\prime}, p\right]$ of $P_{4 m+2}$ each of distance $\left(\frac{1}{3}\right) 2^{-2 m-1}$ from $[h, k]$. Consequently, by Lemma 2.3(c) the iterations $\sigma^{k}([a, p])$ cannot cross the vertical line $[h, k]$. The assertion is proved.

Part (b) follows from Lemma 2.3(c).

Using Proposition 3.1 one easily obtains

Proposition 3.2. $\quad c_{0}=\left(-\frac{1}{3}, \frac{1}{3}\right)$ and $c_{1}=\left(0, \frac{2}{3}\right)$ are cut points of $D$.

Proof. It follows from Proposition 3.1 and Lemma 2.3(c) that only iterations of the three edges $[a, b],[b, d]$, and $[d, e]$ can intersect the line $[f, g]$. Using Proposition 3.1, it suffices to consider iterations of the edge $[b, d]$. Iterating $[b, d]$ four times (see Fig. 3(b)) we get a scaled copy (by a linear factor of $\frac{1}{2}$ ) of Fig. 3(a) near $c_{0}$, with $c_{0}$ as a fixed point. Continuing this process, we see that $c_{0}$ is a cut point of $D$.

To see that $c_{1}$ is also a cut point we need only observe that $c_{1}=\varphi_{2}\left(c_{0}\right)$ and $\varphi_{1}(R)$ intersects only one of the two components of $\varphi_{2}\left(D \backslash\left\{c_{0}\right\}\right)$.

It follows from Proposition 3.2 that $c_{0}$ and $c_{1}$ divide $D$ into three closed sets that are otherwise mutually disjoint. We call the one that contains both $c_{0}$ and $c_{1}$ the central piece of $D$, and denote it throughout the rest of the paper by $C$. We now show that $C$ is a graph self-similar set and find a graph-directed IFS (see [MW] and [E]) defining $C$. 


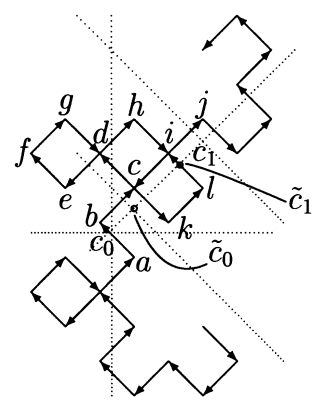

Fig. 4. The parts of $\mathcal{P}_{5}$ that generate the four components of $C$.

Proposition 3.3. $\quad C$ is a graph self-similar set. It is a union of a twin dragon, a contracted copy of itself by a (linear) factor $\frac{1}{2}$, and two contracted copies of itself by a factor of $\frac{1}{4}$.

Proof. We examine the subcurve of $\mathcal{P}_{5}$ whose iterations generate $C$ (see Fig. 4). By comparing with $\mathcal{P}_{2}$, we see that iterations of the subcurve $[e, f] \cup[f, g] \cup[g, d] \cup[d, h]$ converge to a contracted Heighway dragon (by a linear factor of $\frac{1}{4}$ ). Similarly, iterations of $[c, d] \cup[d, e] \cup[h, i] \cup[i, c]$ yield another Heighway dragon of the same size but rotated by an angle $\pi$. The union of these two Heighway dragons forms a twin dragon (see [E]). See Fig. 5.

Define $\tilde{c}_{0}=c_{0}+2^{-3 / 2}(1,1)$ and $\tilde{c}_{1}=c_{1}-2^{-3 / 2}(1,1)$. Comparing $\mathcal{P}_{5}$ with $\mathcal{P}_{1}$ shows that the portion of $\sigma^{\infty}([a, b] \cup[b, c])$ between $c_{0}$ and $\tilde{c}_{0}$ is a contracted copy of $C$. Similarly, the portion of $\sigma^{\infty}([i, l] \cup[i, j])$ between $c_{1}$ and $\tilde{c}_{1}$ is also a contracted copy of $C$. Both copies are contracted by a factor of $\frac{1}{4}$.

Lastly, comparing $\mathcal{P}_{5}$ with $\mathcal{P}_{3}$ shows that the portion of $\sigma^{\infty}([b, c] \cup[c, k] \cup[k, l] \cup[l, i])$ between $\tilde{c}_{0}$ and $\tilde{c}_{1}$ is a contracted copy of $C$ by a linear factor of $\frac{1}{2}$.

We now give a precise description of the graph-directed IFS defining $C$. Throughout

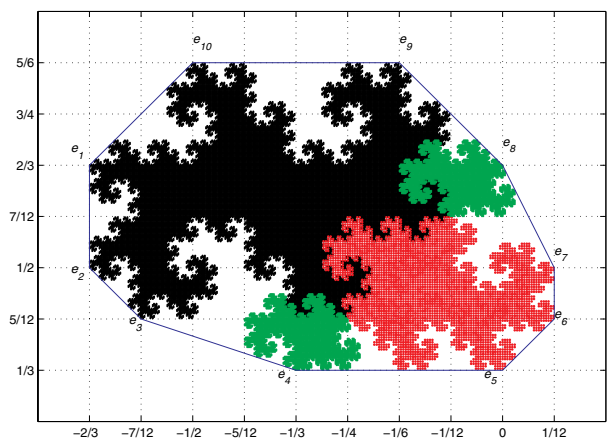

Fig. 5. Figure illustrating the four seed sets of the central piece $C$ together with all the extreme points $e_{1}, \ldots, e_{10}$ and the convex hull $H$. 
the rest of the paper, $G_{1}$ denotes the twin dragon, $G_{2}$ denotes the $\frac{1}{2}$-contracted copy of $C$, and $G_{3}$ and $G_{4}$ denote respectively the lower and upper $\frac{1}{4}$-contracted copies of $C$. $C$ is a graph self-similar set with $G_{1}, G_{2}, G_{3}, G_{4}$ as seed sets. The similitudes are specified as follows: $\psi_{1}, \psi_{2}: G_{1} \rightarrow G_{1}, \psi_{3}: G_{i} \rightarrow G_{2}$ with $i=1,2,3,4, \psi_{4}: G_{2} \rightarrow G_{3}$, and $\psi_{5}: G_{2} \rightarrow G_{4}$, where

$$
\begin{array}{ll}
\psi_{1}(x)=\frac{1}{\sqrt{2}} R\left(\frac{\pi}{4}\right) x+\left[\begin{array}{l}
0 \\
\frac{1}{2}
\end{array}\right], & \psi_{2}(x)=\frac{1}{\sqrt{2}} R\left(\frac{\pi}{4}\right) x+\left[\begin{array}{c}
\frac{1}{4} \\
\frac{1}{2}
\end{array}\right], \\
\psi_{3}(x)=\frac{1}{2} R(\pi) x+\left[\begin{array}{c}
-\frac{1}{4} \\
\frac{3}{4}
\end{array}\right], & \psi_{4}(x)=\frac{1}{2} R(\pi) x+\left[\begin{array}{c}
-\frac{3}{8} \\
\frac{5}{8}
\end{array}\right], \\
\psi_{5}(x)=\frac{1}{2} R(\pi) x+\left[\begin{array}{c}
-\frac{1}{8} \\
\frac{7}{8}
\end{array}\right] . &
\end{array}
$$

Let $H$ denote the convex hull of $C$. Since the $\psi_{i}$ defining $C$ have different linear parts, it does not seem possible to modify the technique in [SW] to determine $H$. We use a more elementary method instead. Let $e_{i}, i=1, \ldots, 10$, denote the points (see Figure 5)

$$
\begin{array}{llll}
e_{1}=\left(-\frac{2}{3}, \frac{2}{3}\right), & e_{2}=\left(-\frac{2}{3}, \frac{1}{2}\right), & e_{3}=\left(-\frac{7}{12}, \frac{5}{12}\right), & e_{4}=\left(-\frac{1}{3}, \frac{1}{3}\right), \\
e_{5}=\left(0, \frac{1}{3}\right), & e_{6}=\left(-\frac{1}{12}, \frac{5}{12}\right), & e_{7}=\left(\frac{1}{12}, \frac{1}{2}\right), & e_{8}=\left(0, \frac{2}{3}\right), \\
e_{9}=\left(-\frac{1}{6}, \frac{5}{6}\right), & e_{10}=\left(-\frac{1}{2}, \frac{5}{6}\right) . & &
\end{array}
$$

Proposition 3.4. $E:=\left\{e_{i}\right\}_{i=1}^{10}$ is the set of all extreme points of $C$. Therefore $H$ is the convex hull of $E$ and is a 10-sided polygon.

Proof. Because all extreme points of $D$ are known (see [BP]), it is easy to see that $E \subset$ $C$. It suffices to show that each line segment $\left[e_{i}, e_{i+1}\right]$ belongs to $\partial H$ for $i=1, \ldots, 10$ (with $e_{11}:=e_{1}$ ). $\left[e_{10}, e_{1}\right] \subseteq \partial H$ because it is a side in the boundary of the convex hull of $D$. Similarly, $\left[e_{1}, e_{2}\right] \subseteq \partial H$. Since $e_{3}, e_{5}, e_{6}$ lie on the boundary of the convex hull of $\varphi_{2}(D),\left[e_{2}, e_{3}\right] \cup\left[e_{5}, e_{6}\right] \subseteq \partial H$. It follows from Propositions 3.1 and 3.2 that $\left[e_{4}, e_{5}\right] \cup\left[e_{8}, e_{9}\right] \subseteq \partial H$.

Now, let $b$ denote the extreme point $\left(\frac{1}{3}, \frac{2}{3}\right)$ of $D$. Since $\left[e_{9}, e_{10}\right]=\varphi_{2}\left(\left[e_{5}, b\right]\right)$ and $\left[e_{5}, b\right]$ belongs to the boundary of the convex hull of $\varphi_{2}(D)$, we conclude that $\left[e_{9}, e_{10}\right] \subseteq$ $\partial H$. Similarly, $\left[e_{6}, e_{7}\right]=\varphi_{2}\left(\left[e_{2}, e_{3}\right]\right)$ and thus $\left[e_{6}, e_{7}\right] \subseteq \partial H$.

Lastly, among $G_{i}, i=1, \ldots, 4$, the open line segment $\left(e_{3}, e_{4}\right)$ only intersects $G_{3}=$ $\varphi_{4}(C)$, which is a contraction of $C$ (with no rotation) with $e_{4}$ as a fixed point. Thus $\left[e_{3}, e_{4}\right] \subseteq \partial H$. The same argument shows that $\left[e_{7}, e_{8}\right] \subseteq \partial H$.

Some additional properties of $C$ will be proved after all cut points of $D$ are determined (see Proposition 5.3). 


\section{Interior of the Central Piece}

We will prove the proposition that $C^{\circ}$ is arcwise connected. It follows from the proof of Proposition 3.1 that, for each $n$, there exists a unique edge $\mathcal{E}_{n} \in E\left(\mathcal{P}_{n}\right)$ intersecting the line $y=\frac{1}{3}$. Similarly, there exists a unique edge $\mathcal{F}_{n} \in E\left(\mathcal{P}_{n}\right)$ intersecting the line $y=-x+\frac{2}{3}$. In fact, $\left\{c_{0}\right\}=\lim _{n \rightarrow \infty} \mathcal{E}_{n}$ and $\left\{c_{1}\right\}=\lim _{n \rightarrow \infty} \mathcal{F}_{n}$ in the Hausdorff metric.

Definition 4.1. Three consecutive edges of $\mathcal{P}_{n}$ are said to be dependent if there exists a square (not necessarily a small square of $\mathcal{P}_{n}$ ) having these three edges as its edges. Three consecutive edges of $P_{n}$ are said to be independent if they are not dependent.

For $n \geq 5$, let $u_{n}$ denote the vertex of the small square in $\mathcal{P}_{n}$ which is first visited by the principal path right after passing the edge $\mathcal{E}_{n}$ and let $v_{n}$ denote the vertex of the small square in $\mathcal{P}_{n}$ which is last visited by the principal path right before going to $\mathcal{F}_{n}$; see Fig. 6. Let $\mathcal{D}_{n}$ denote the part of $\mathcal{P}_{n}$ between $u_{n}$ and $v_{n}$. Let $\mathcal{U}_{n}$ (resp. $\mathcal{V}_{n}$ ) be the union of $\mathcal{E}_{n}$ (resp. $\mathcal{F}_{n}$ ) with its two neighboring edges. Claim 4.1 justifies the existence of $u_{n}, v_{n}, \mathcal{U}_{n}, \mathcal{V}_{n}$.

Claim 4.1. For any $n \geq 5$ the points $u_{n}$ and $v_{n}$ are well defined. Moreover, both $\mathcal{U}_{n}$ and $\mathcal{V}_{n}$ consist of three independent edges.

Proof. Clearly the claim is true for $n=5$. We will only prove it for $u_{n}$ and $\mathcal{U}_{n}$. Assume that it holds for some $n \geq 5$. We notice by Observation 2.1(a) that each of the two endpoints of $\mathcal{U}_{n}$ must intersect four edges of $\mathcal{P}_{n}$. Therefore there are only two possible patterns for iterating $\mathcal{U}_{n}$; both are shown in Fig. 7.

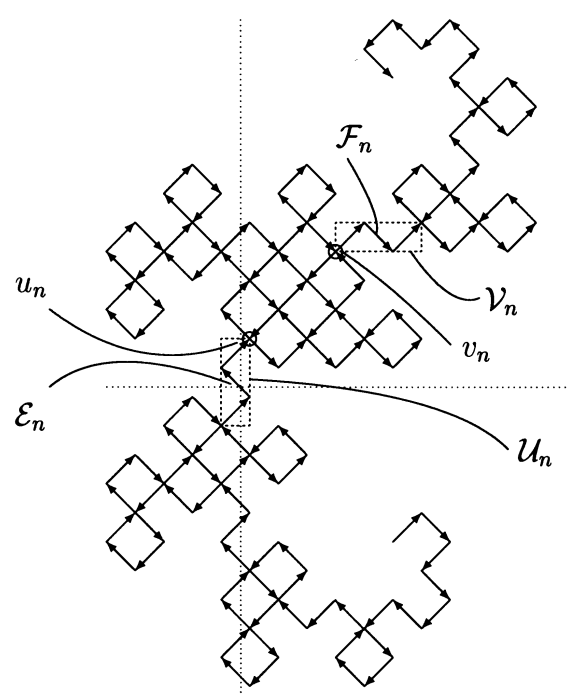

Fig. 6. The vertices $u_{n}, v_{n}$ and the edges $\mathcal{E}_{n}, \mathcal{F}_{n}$, drawn for $n=7$. The subcurves $\mathcal{U}_{n}$ and $\mathcal{V}_{n}$ are enclosed by dashed boxes. The intersection of the dotted lines is the cut point $c_{0}$. $\mathcal{D}_{n}$ is the part "between" $u_{n}$ and $v_{n}$. 


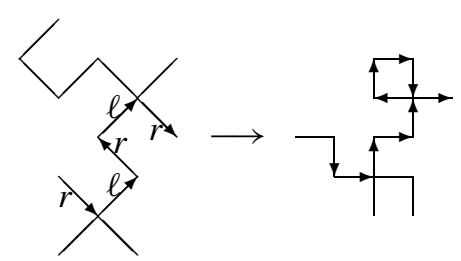

Case 1

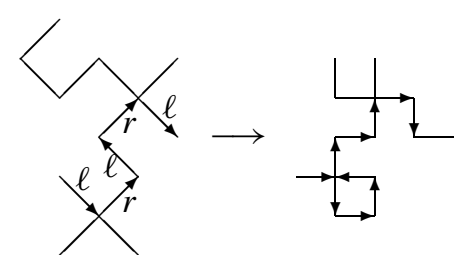

Case 2

Fig. 7. The only two possible ways of iterating $\mathcal{U}_{n}$ and its immediate neighboring edges. Edges labeled $\ell$ or $r$ belong to $E_{\ell}\left(\mathcal{P}_{n}\right)$ or $E_{\mathrm{r}}\left(\mathcal{P}_{n}\right)$, respectively.

In both cases, among the six edges of $\sigma\left(\mathcal{U}_{n}\right)$, three are independent and three are dependent. The three independent edges form the subcurve $\mathcal{U}_{n+1}$ in $\mathcal{P}_{n+1}$, and the three dependent ones are joined with $\sigma\left(\mathcal{P}_{n}\right)$ to create a new small square of $\mathcal{P}_{n+1}$. The claim is proved.

Claim 4.2. For any $n \geq 5$ the subcurve $\mathcal{D}_{n}$ consists only of connected small squares.

Proof. Note that the claim is true for $n=5$. To see that it holds for all $n \geq 5$ let $\mathcal{Q}\left(\sigma\left(\mathcal{U}_{n}, \mathcal{D}_{n}\right)\right)$ denote the new small square of $\mathcal{P}_{n+1}$ created by joining $\sigma\left(\mathcal{U}_{n}\right)$ with $\sigma\left(\mathcal{D}_{n}\right)$. Define $\mathcal{Q}\left(\sigma\left(\mathcal{V}_{n}, \mathcal{D}_{n}\right)\right)$ analogously. Then

$$
\mathcal{D}_{n+1}=\sigma\left(\mathcal{D}_{n}\right) \cup \mathcal{Q}\left(\sigma\left(\mathcal{U}_{n}, \mathcal{D}_{n}\right)\right) \cup \mathcal{Q}\left(\sigma\left(\mathcal{V}_{n}, \mathcal{D}_{n}\right)\right) .
$$

By the proof of Claim 4.1 the subcurve $\mathcal{Q}\left(\sigma\left(\mathcal{U}_{n}, \mathcal{D}_{n}\right)\right) \cup \mathcal{Q}\left(\sigma\left(\mathcal{V}_{n}, \mathcal{D}_{n}\right)\right)$ consists only of at most two small squares of $\mathcal{D}_{n+1}$; see Figure 7. Consequently, to complete the proof of the claim by induction it suffices to observe that if a subcurve $\mathcal{C} \subset \mathcal{P}_{n}$ consists only of connected small squares, then so does $\sigma(\mathcal{C})$.

Lemma 4.3. Let $\mathcal{R}, \mathcal{S}, \mathcal{T}$ be distinct small squares in $\mathcal{P}_{n}$ such that $\mathcal{R}$ and $\mathcal{T}$ are adjacent to $\mathcal{S}$. Let $a$ and $b$ be the vertices of $\mathcal{S}$ intersecting $\mathcal{R}$ and $\mathcal{T}$, respectively. Then the line segment $[a, b]$ is contained in $D^{\circ}$.

Proof. Using Observation 2.1(a) we can assume that $\mathcal{R}$ and $\mathcal{T}$ are connected to $\mathcal{S}$ as shown in Cases 1 and 2 of Fig. 8. There are only four possible configurations in each case. Let $\mathcal{L}:=\mathcal{R} \cup \mathcal{S} \cup \mathcal{T}$.

Case 1: $a$ and $b$ are diagonally opposite vertices of $\mathcal{S}$. In (I), iterating $\mathcal{L}$ five times generates a circle $\tilde{\mathcal{C}}_{n+5}$ in $\mathcal{P}_{n+5}$ enclosing $[a, b]$ properly and is strictly inside a circle $\mathcal{C}_{n+5}$ in $\mathcal{P}_{n+5}$. (The iteration is a little tedious but straightforward.) Moreover, each edge of $\mathcal{M}_{n}$ enclosed by $\mathcal{C}_{n+5}$ is an edge of $\mathcal{P}_{n}$. Hence by Lemma $2.5, \Omega\left(\tilde{\mathcal{C}}_{n+5}\right) \subseteq D^{\circ}$. In (II) we iterate $\mathcal{L}$ twice and let $c$ be the mid-point between $a$ and $b$. Note that now $a, c$, and $b$ are vertices in $\mathcal{P}_{n+2}$ exactly as in (I). Hence $[a, c] \cup[c, b] \subseteq D^{\circ}$, and thus $[a, b] \subseteq D^{\circ}$. Upon iteration (III) and (IV) yield the same subcurves as (I) and (II) do, respectively, with the directions of the edges being reversed. Thus the lemma holds in this case. 


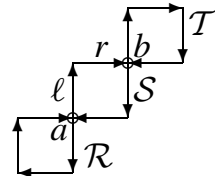

(I)

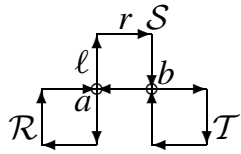

(V)

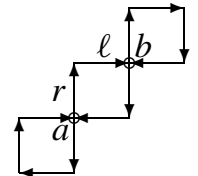

(II)

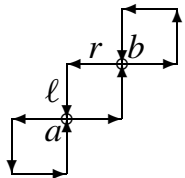

(III)

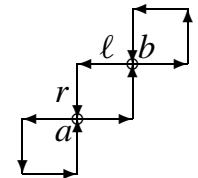

(IV)

Case 1

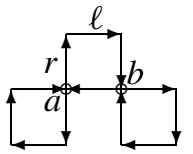

(VI)

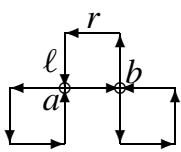

(VII)

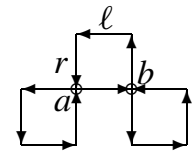

(VIII)

Case 2

Fig. 8. Case 1. The four configurations in which $a$ and $b$ are diagonally opposite. Case 2. $a$ and $b$ are not diagonally opposite.

Case 2: $a$ and $b$ are not diagonally opposite. In (V), upon iterating $\mathcal{L}$ once, $a$ and $b$ become diagonally opposite, and we can apply the same argument of Case 1. In (VI) we iterate $\mathcal{L}$ twice and let $c$ be the mid-point between $a$ and $b$. Then by (V), $[a, c] \cup[c, b] \subseteq D^{\circ}$. Iterations of (VII) and (VIII) yield the same subcurves as those generated by (V) and (VI), respectively. The lemma is proved.

Proposition 4.4. $\quad C^{\circ}$ is arcwise connected.

Proof. Observe that $C=\lim _{k \rightarrow \infty} \mathcal{D}_{n}$ in the Hausdorff metric. Let $a, b \in C^{\circ}$. Since $a$ and $b$ lie in the closure of $\bigcup_{n=0}^{\infty} V\left(\mathcal{D}_{n}\right)$, using Claim 4.2 we can choose two sequences $\left\{a_{n}\right\}$ and $\left\{b_{n}\right\}$ of vertices of connected small squares in $\mathcal{D}_{n}$ which converge to $a$ and $b$, respectively. Using Lemma 4.3 and taking subsequences of $\left\{a_{n}\right\}$ and $\left\{b_{n}\right\}$ if necessary, we can find two curves $\gamma_{a}, \gamma_{b}:[0,1] \rightarrow D^{\circ}$ connecting $\left\{a_{n}\right\}$ and $\left\{b_{n}\right\}$, respectively. More precisely,

$$
\gamma_{a}(1 / n)=a_{n}, \quad \gamma_{a}(0)=a
$$

and

$$
\gamma_{b}(1 / n)=b_{n}, \quad \gamma_{b}(0)=b .
$$

Now we can use the same argument to construct a curve in $D^{\circ}$ connecting $a_{1}$ and $b_{1}$.

It follows from the tiling property that any component of the interior of a connected reptile or self-affine tile in $\mathbb{R}^{2}$ is an open topological disk (see [BW] and [LRT]). Thus Proposition 4.4 yields

Corollary 4.5. $\quad C^{\circ}$ is an open topological disk. 
We conclude this section by strengthening Lemma 2.5 on the central piece $C$.

\section{Lemma 4.6.}

(a) Let $\mathcal{C}, \tilde{\mathcal{C}}$ be circles in $\mathcal{D}_{n}$ with $\tilde{\mathcal{C}}$ lying strictly inside $\mathcal{C}$. Then $\Omega(\tilde{\mathcal{C}}) \subseteq C^{\circ}$.

(b) Let $\mathcal{C}$ be a circle in $\mathcal{M}_{n}$ such that $\mathcal{C} \subset C^{\circ}$. Then all edges in $E\left(\mathcal{M}_{n}\right)$ which lie inside $\mathcal{C}$ belong to $E\left(\mathcal{D}_{n}\right)$.

Proof. We prove (a); the proof of (b) is similar. Using Lemma 2.5 it suffices to show that each edge of $\mathcal{M}_{n}$ inside $\mathcal{C}$ is an edge in $E\left(\mathcal{P}_{n}\right)$. Suppose on the contrary that there exists an edge $\mathcal{E} \in E\left(\mathcal{M}_{n}\right) \backslash E\left(\mathcal{P}_{n}\right)$ inside $\mathcal{C}$.

Case 1: $\mathcal{C}$ encloses a vertex $v \in V\left(\mathcal{M}_{n}\right) \backslash V\left(\mathcal{P}_{n}\right)$. By Lemma 2.3(a), $v \notin C$. However, by Claim 4.2 and Lemma 4.3, there exists a curve $\gamma \subseteq C^{\circ}$ enclosing $v$, contradicting Corollary 4.5 .

Case 2: Each vertex enclosed by $\mathcal{C}$ is in $V\left(\mathcal{P}_{n}\right)$. By Observation 2.1(a), each endpoint of $\mathcal{E}$ must be connected to another edge in $E\left(\mathcal{M}_{n}\right) \backslash E\left(\mathcal{P}_{n}\right)$. Denote them by $\mathcal{F}$ and $\mathcal{G}$. First, observe that $\mathcal{E}, \mathcal{F}, \mathcal{G}$ must be dependent. Otherwise, Proposition 2.2 would force $\mathcal{E}$ to be an edge in $\mathcal{P}_{n}$ connecting two parallel and noncollinear edges in $\mathcal{P}_{n}$. Next, by Proposition 2.2 again, the fourth edge of the small square formed by $\mathcal{E}, \mathcal{F}, \mathcal{G}$ must also be an edge of $E\left(\mathcal{M}_{n}\right) \backslash E\left(\mathcal{P}_{n}\right)$. Now, a similar argument as that in Case 1, with $v$ replaced by the center of the small square, yields a contradiction.

\section{Proof of the Main Results}

The following key lemma determines the composition and the cut points of $D$.

Lemma 5.1. The Heighway dragon $D$ is the union of the geometrically similar contracted copies $C_{n}, n \in \mathbb{Z}$, of $C$, together with $C_{-\infty}$ and $C_{\infty}$. Moreover, for each $n \in \mathbb{Z}$, $C_{n}$ has nonempty intersection only with $C_{n-1}$ and $C_{n+1}$. More precisely,

$$
\begin{aligned}
D & =\bigcup_{n \in \mathbb{Z} \cup\{-\infty, \infty\}} C_{n} \quad \text { and for each } n \in \mathbb{Z}, \\
C_{n} \cap C_{m} & = \begin{cases}\left\{c_{n}\right\}, & m=n-1, \\
\left\{c_{n+1}\right\}, & m=n+1, \\
\emptyset, & \text { otherwise. }\end{cases}
\end{aligned}
$$

Proof. By Proposition 3.2, the cut points $c_{0}$ and $c_{1}$ divide $D$ into three pieces, i.e.,

$$
D=D_{-1} \cup C \cup D_{1}, \quad C \cap D_{-1}=\left\{c_{0}\right\}, \quad C \cap D_{1}=\left\{c_{1}\right\},
$$

where $D_{-1}:=\overline{\varphi_{1}(D) \backslash C}$ and $D_{1}:=\overline{\varphi_{2}(D) \backslash C}$. 
We claim that for each $k \geq 1$,

$$
\begin{aligned}
D= & \left(\bigcup_{n=-k}^{k} C_{n}\right) \cup\left[\varphi_{1}^{k}\left(D_{-1}\right) \cup \varphi_{2} \circ \varphi_{1}^{k}\left(D_{-1}\right)\right], \\
& C_{-k} \cap \varphi_{1}^{k}\left(D_{-1}\right)=\left\{c_{-k}\right\}, \\
C_{k} \cap \varphi_{2} \circ \varphi_{1}^{k}\left(D_{-1}\right)= & \left\{c_{k+1}\right\}, \quad\left(\bigcup_{n=-(k-1)}^{k-1} C_{n}\right) \cap\left[\varphi_{1}^{k}\left(D_{-1}\right) \cup \varphi_{2} \circ \varphi_{1}^{k}\left(D_{-1}\right)\right]=\emptyset ;
\end{aligned}
$$

moreover, $\left\{C_{n}:-k<n<k\right\}$ satisfies (5.1).

Substituting the first equality in (5.2) into the self-similar identity (1.2) and comparing both sides leads to

$$
C=\varphi_{1}\left(D_{1}\right) \cup \varphi_{2}(C) \cup \varphi_{2}\left(D_{1}\right), \quad D_{1}=\varphi_{2}\left(D_{-1}\right), \quad D_{-1}=\varphi_{1}\left(D_{-1}\right) \cup \varphi_{1}(C) .
$$

Using these equalities, we can rewrite $D$ as

$$
D=\left[C_{-1} \cap C \cup C_{1}\right] \cup\left[\varphi_{1}\left(D_{-1}\right) \cup \varphi_{2} \circ \varphi_{1}\left(D_{-1}\right)\right]
$$

with

$$
\begin{aligned}
C_{-1} \cap \varphi_{1}\left(D_{-1}\right) & =\varphi_{1}\left(C \cap D_{-1}\right)=\left\{\varphi_{1}\left(c_{0}\right)\right\}=\left\{c_{-1}\right\}, \\
C_{1} \cap \varphi_{2} \circ \varphi_{1}\left(D_{-1}\right) & =\varphi_{2} \circ \varphi_{1}\left(C \cap D_{-1}\right)=\left\{\varphi_{2} \circ \varphi_{1}\left(c_{0}\right)\right\}=\left\{c_{2}\right\} .
\end{aligned}
$$

It is now easy to see that the claim holds for $k=1$. It follows by induction that it holds for all $k \geq 1$. The lemma follows immediately from the claim.

We can get analogous results for the convex hulls $H_{n}$. We label the four boundary sides of $H_{n}$ that intersect the cut points. Define

$$
\begin{array}{lll}
E_{0}^{-}:=\left[e_{3}, e_{4}\right], & E_{0}^{+}:=\left[e_{4}, e_{5}\right], & E_{0}:=E_{0}^{-} \cup E_{0}^{+}, \\
F_{0}^{-}:=\left[e_{7}, e_{8}\right], & F_{0}^{+}:=\left[e_{8}, e_{9}\right], & F_{0}:=F_{0}^{-} \cup F_{0}^{+},
\end{array}
$$

and for all $n \geq 1$ define

$$
\begin{array}{rlrl}
E_{-n}^{-} & :=\varphi_{1}^{n}\left(E_{0}^{-}\right), & E_{-n}^{+}:=\varphi_{1}^{n}\left(E_{0}^{+}\right), & E_{-n}:=\varphi_{1}^{n}\left(E_{0}\right), \\
E_{n}^{-}:=\varphi_{2} \circ \varphi_{1}^{n-1}\left(E_{0}^{-}\right), & E_{n}^{+}:=\varphi_{2} \circ \varphi_{1}^{n-1}\left(E_{0}^{+}\right), & E_{n}:=\varphi_{2} \circ \varphi_{1}^{n-1}\left(E_{0}\right), \\
F_{n}^{-}:=\varphi_{2} \circ \varphi_{1}^{n-1}\left(F_{0}^{-}\right), & F_{n}^{+}:=\varphi_{2} \circ \varphi_{1}^{n-1}\left(F_{0}^{+}\right), & F_{n}:=\varphi_{2} \circ \varphi_{1}^{n-1}\left(F_{0}\right) .
\end{array}
$$

Note that $F_{0}^{-}=E_{1}^{-}$and $F_{0}^{+}=E_{1}^{+}$. Part (a) of the following lemma strengthens Lemma 5.1.

\section{Lemma 5.2.}

(a) For each $n \in \mathbb{Z}, H_{n}$ has nonempty intersection only with $H_{n-1}$ and $H_{n+1}$. More precisely, for all $m, n \in \mathbb{Z}$,

$$
H_{n} \cap H_{m}= \begin{cases}\left\{c_{n}\right\}, & m=n-1, \\ \left\{c_{n+1}\right\}, & m=n+1, \\ \emptyset, & \text { otherwise. }\end{cases}
$$


(b) For all $n \geq 0$,

$$
\begin{gathered}
H_{-n} \cap H_{-(n+1)}=E_{-n} \cap F_{-(n+1)}=E_{-n}^{-} \cap F_{-(n+1)}^{-}=E_{-n}^{+} \cap F_{-(n+1)}^{+}=\left\{c_{-n}\right\}, \\
H_{n} \cap H_{n+1}=E_{n+1} \cap F_{n+1}=E_{n+1}^{-} \cap F_{n+1}^{-}=E_{n+1}^{+} \cap F_{n+1}^{+}=\left\{c_{n+1}\right\} .
\end{gathered}
$$

Moreover, at the intersection $c_{-n}, E_{-n}^{-} \cup F_{-(n+1)}^{-}$and $E_{-n}^{+} \cup F_{-(n+1)}^{+}$are line segments; at the intersection $c_{n+1}, E_{n+1}^{-} \cup F_{-(n+1)}^{-}$and $E_{n+1}^{+} \cup F_{n+1}^{+}$are line segments.

Proof. (a) The proof is similar to that of Lemma 5.1. It follows from Propositions 3.1 and 3.2 that

$$
\begin{aligned}
H_{0} & \cap\left(\bigcup_{n \leq-1} H_{n}\right)=H_{0} \cap H_{-1}=\left\{c_{0}\right\}, \\
H_{0} \cap\left(\bigcup_{n \geq 1} H_{n}\right) & =H_{0} \cap H_{1}=\left\{c_{1}\right\}, \\
H_{0} \cap\left(\bigcup_{|n| \geq 2} H_{n}\right) & =\emptyset \\
\left(\bigcup_{n=1}^{\infty} H_{-n}\right) & \cap\left(\bigcup_{n=1}^{\infty} H_{n}\right)=\emptyset .
\end{aligned}
$$

Applying $\varphi_{1}$ to (5.3), (5.5), and $\varphi_{2} \circ \varphi_{1}$ to (5.4), (5.5), and then using (5.6), we can prove by induction that for all $k \geq 1$,

$$
\begin{aligned}
H_{-k} \cap\left(\bigcup_{n \leq-(k+1)} H_{n}\right) & =H_{-k} \cap H_{-(k+1)}=\left\{c_{-k}\right\}, \\
H_{k} & \cap\left(\bigcup_{n \geq k+1} H_{n}\right)=H_{k} \cap H_{k+1}=\left\{c_{k+1}\right\}, \\
\left(\bigcup_{|n| \leq k} H_{n}\right) \cap\left(\bigcup_{|n| \geq k+2} H_{n}\right) & =\emptyset,
\end{aligned}
$$

from which the assertion follows.

(b) The equalities in (b) follow from (a). To prove the last assertion, we first note that the slope of $F_{0}^{+}$is -1 and therefore $F_{-1}^{+}=\varphi_{1}\left(F_{0}^{+}\right)$is horizontal. Thus, $E_{0}^{+}$and $F_{-1}^{+}$are collinear. A similar argument shows that $E_{0}^{-}$and $F_{-1}^{-}$are collinear. The assertion now follows from the fact that for all $n \geq 1, \varphi_{1}^{n}$ and $\varphi_{2} \circ \varphi_{1}^{n-1}$ are similitudes.

Proof of Theorem 1.3. Lemma 5.1 implies that all $c_{n}, n \in \mathbb{Z}$, are cut points of $D$. By Corollary 4.5, these are the only cut points of $D$. Hence, (a) and (b) follow from Lemma 5.1. Part (c) follows from Lemma 5.2. The two exact formulas in (d) can be proved directly by induction. This completes the proof. 
For any measurable subset $E \subseteq \mathbb{R}^{2}$, we let $m(E)$ denote the area (i.e., Lebesgue measure) of $E$. It is known that $m(D)=\frac{1}{2}$ (see [BP]). We use this result to find the area $C$ and show that the graph-directed IFS defining $C$ satisfies the graph open set condition (see, e.g., [S]). It is well known that the maps $\psi_{1}, \psi_{2}$ (see Section 3) defining the twin dragon $G_{1}$ satisfy the open set condition.

Proposition 5.3. The area of $C$ is $m(C)=2 m(D) / 5=\frac{1}{5}$. Moreover, the graphdirected IFS defining $C$ satisfies the graph open set condition.

Proof. By summing up the areas of the $C_{n}$ in Lemma 5.1, we have

$$
m(D)=\sum_{n=0}^{\infty}\left(\frac{1}{2}\right)^{n} m(C)+\sum_{n=2}^{\infty}\left(\frac{1}{2}\right)^{n} m(C)=\frac{5 m(C)}{2},
$$

which yields the area $m(C)$. This and Proposition 3.3 together imply that the sum of the areas of the fours seed sets $G_{1}, G_{2}, G_{3}, G_{4}$ making up $C$ is equal to $m(C)$. Thus the four seed sets must be essentially disjoint, and therefore the graph open set condition holds.

We now prove that $C$ is a closed topological disk. Let $T$ be a self-similar or self-affine set defined by an IFS satisfying the open set condition. It is known that if $T^{\circ} \neq \varnothing$ and $T^{\circ}$ is connected, then $T$ is a topological disk (see [LRT] and [BW]). Unfortunately the analogous statement for graph self-similar sets is false and the proof in [LRT] cannot be generalized. As a counterexample, divide any square into nine equal subsquares, and let $T$ be the compact set obtained by removing the central subsquare and any of the corner ones.

Our proof uses an argument of Bandt and Wang. The key is to show that each point $x \in \partial C$ is simple (see Chapter 14 of $[\mathrm{R}]$ ). To each sequence $\left\{x_{k}\right\} \subset C^{\circ}$ converging to $x$, we will construct a curve in $\gamma \subset C^{\circ}$ connecting the $x_{k}$ which also converges to $x$, i.e.,

$$
\sup \left\{\|y-x\|: y \in \gamma_{x_{k}}\right\} \rightarrow 0 \quad \text { as } \quad k \rightarrow \infty
$$

where $\gamma_{x_{k}}$ denotes the "tail" of $\gamma$ starting from $x_{k}$. This will show that $x$ is simple.

For each $n$ sufficiently large, let $\mathcal{D}_{n}$ be defined as in Section 4 . We first prove:

Lemma 5.4. For any two sequences $\left\{a_{n}\right\}$ and $\left\{b_{n}\right\}$ of vertices of $\mathcal{D}_{n}$ we have

$$
\left\|a_{n}-b_{n}\right\| \rightarrow 0 \quad \text { implies } \operatorname{diam}\left(\mathcal{L}\left[a_{n}, b_{n}\right]\right) \rightarrow 0,
$$

where $\mathcal{L}[a, b]$ denotes a shortest subcurve of $\mathcal{D}_{n}$ from a to $b$.

Proof. Suppose the lemma is false. Then we can find two subsequences $\left\{a_{n_{k}}\right\}$ and $\left\{b_{n_{k}}\right\}$ of vertices of $\mathcal{D}_{n_{k}}$ such that

$$
\begin{aligned}
& \lim _{k \rightarrow \infty} a_{n_{k}}=\lim _{k \rightarrow \infty} b_{n_{k}}=u \in D, \quad \text { and } \\
& \operatorname{diam}\left(\mathcal{L}\left[a_{n_{k}}, b_{n_{k}}\right]\right)>\delta>0 \quad \text { for every } k .
\end{aligned}
$$


Fix $k_{0}$ such that

$$
2^{-n_{k_{0}} / 2}<\delta / 7
$$

For each $k \geq k_{0}$ we choose edges $\left[a_{k}, a_{k}^{\prime}\right]$ and $\left[b_{k}, b_{k}^{\prime}\right]$ of $\mathcal{D}_{n_{k_{0}}}$ such that

$$
a_{n_{k}} \in \sigma^{n_{k}-n_{k_{0}}}\left(\left[a_{k}, a_{k}^{\prime}\right]\right) \quad \text { and } \quad b_{n_{k}} \in \sigma^{n_{k}-n_{k_{0}}}\left(\left[b_{k}, b_{k}^{\prime}\right]\right) .
$$

Observe that $\left[a_{k}, a_{k}^{\prime}\right]$ and $\left[b_{k}, b_{k}^{\prime}\right]$ depend on $k$. However, since the number of edges of $\mathcal{D}_{n_{k_{0}}}$ is finite, by passing to a subsequence if necessary, we may assume that

$$
\left[a_{k}, a_{k}^{\prime}\right]=\left[a_{k_{0}}, a_{k_{0}}^{\prime}\right] \quad \text { and } \quad\left[b_{k}, b_{k}^{\prime}\right]=\left[b_{k_{0}}, b_{k_{0}}^{\prime}\right] \quad \text { for every } k \text {. }
$$

By Lemma 2.3(c) we have, for every $m \geq 0$,

$$
\begin{aligned}
& \operatorname{diam}\left(\sigma^{m}\left(\left[a_{k_{0}}, a_{k_{0}}^{\prime}\right]\right)\right) \leq \frac{\sqrt{13}}{2}\left\|a_{k_{0}}-a_{k_{0}}^{\prime}\right\| \leq 2\left(2^{-n_{k_{0}} / 2}\right) ; \\
& \operatorname{diam}\left(\sigma^{m}\left(\left[b_{k_{0}}, b_{k_{0}}^{\prime}\right]\right)\right) \leq 2\left(2^{-n_{k_{0}} / 2}\right) .
\end{aligned}
$$

From (5.7) we get

$$
u \in \sigma^{\infty}\left(\left[a_{k_{0}}, a_{k_{0}}^{\prime}\right]\right) \cap \sigma^{\infty}\left(\left[b_{k_{0}}, b_{k_{0}}^{\prime}\right]\right) .
$$

Therefore by Proposition 2.4, for $k \geq k_{0}$ there is a subcurve $\mathcal{L}_{k} \subset \mathcal{D}_{n_{k}}$ consisting of at most three edges connecting $\sigma^{n_{k}-n_{k_{0}}}\left(\left[a_{k_{0}}, a_{k_{0}}^{\prime}\right]\right)$ with $\sigma^{n_{k}-n_{k_{0}}}\left(\left[b_{k_{0}}, b_{k_{0}}^{\prime}\right]\right)$. Hence the set

$$
S_{k}:=\sigma^{n_{k}-n_{k_{0}}}\left(\left[a_{k_{0}}, a_{k_{0}}^{\prime}\right]\right) \cup \sigma^{n_{k}-n_{k_{0}}}\left(\left[b_{k_{0}}, b_{k_{0}}^{\prime}\right]\right) \cup \mathcal{L}_{k}
$$

contains a subcurve connecting $a_{n_{k}}$ with $b_{n_{k}}$, and from (5.9) we get

$$
\begin{aligned}
\operatorname{diam}\left(\mathcal{L}\left[a_{n_{k}}, b_{n_{k}}\right]\right) & \leq \operatorname{diam}\left(S_{k}\right) \\
& \leq \operatorname{diam}\left(\sigma^{n_{k}-n_{k_{0}}}\left(\left[a_{k_{0}}, a_{k_{0}}^{\prime}\right]\right)\right)+\operatorname{diam}\left(\sigma^{n_{k}-n_{k_{0}}}\left(\left[b_{k_{0}}, b_{k_{0}}^{\prime}\right]\right)\right)+\operatorname{diam}\left(\mathcal{L}_{k}\right) \\
& \leq 2\left(2^{-n_{k_{0}} / 2}\right)+2\left(2^{-n_{k_{0}} / 2}\right)+3\left(2^{-n_{k} / 2}\right) \leq 7\left(2^{-n_{k_{0}} / 2}\right)<\delta .
\end{aligned}
$$

This contradicts (5.8) and so the lemma is proved.

Theorem 5.5. Each point $x \in \partial C$ is simple. Consequently, $C$ is a closed topological disk.

Proof. Let $\left\{x_{k}\right\}$ be a sequence in $C^{\circ}$ which converges to $x \in \partial C$. Using Corollary 4.5 and Lemma 4.6, we can find a sequence of circles $\mathcal{C}_{n_{k}} \subset \mathcal{D}_{n_{k}}$ such that:

(a) $\left\{\Omega\left(\mathcal{C}_{n_{k}}\right)\right\}$ is an increasing sequence (i.e., $\Omega\left(\mathcal{C}_{n_{k}}\right) \subset \Omega\left(\mathcal{C}_{n_{k+1}}\right)$ for all $k$ ) of closed disks with $\Omega\left(\mathcal{C}_{n_{k}}\right) \subset C^{\circ}$ for every $k$.

(b) $x_{k} \in \Omega\left(\mathcal{Q}_{n_{k}}\right)$ for every $k$, where $\mathcal{Q}_{n_{k}}$ is a small square in $\mathcal{D}_{n_{k}}$ inside $\mathcal{C}_{n_{k}}$.

We first define a curve $\tilde{\gamma}$ connecting the following sequence of points in the given order:

$$
x_{1} \rightarrow b_{1} \rightarrow a_{2} \rightarrow x_{2} \rightarrow b_{2} \rightarrow \cdots \rightarrow a_{k} \rightarrow x_{k} \rightarrow b_{k} \rightarrow a_{k+1} \rightarrow \cdots \rightarrow x,
$$


where $a_{k}$ and $b_{k}$ are vertices of $\mathcal{Q}_{n_{k}}$ which are closest to $\mathcal{Q}_{n_{k-1}}$ and $\mathcal{Q}_{n_{k+1}}$ respectively, the curve $a_{k} \rightarrow x_{k} \rightarrow b_{k}$ is the union of the two line segments $\left[a_{k}, x_{k}\right] \cup\left[x_{k}, b_{k}\right] \subseteq \Omega\left(\mathcal{Q}_{n_{k}}\right)$, and the curve $b_{k} \rightarrow a_{k+1}$ is a shortest subcurve $\mathcal{L}\left[b_{k}, a_{k+1}\right]$ of $\mathcal{D}_{n_{k+1}}$ described by Lemma 5.4. (Observe that both $b_{k}$ and $a_{k+1}$ are vertices of $\mathcal{D}_{n_{k+1}}$.)

Next, note that $\mathcal{L}\left[b_{k}, a_{k+1}\right]$ need not lie in $C^{\circ}$. However, from Claim 4.2 and Lemma 4.3 there exists a curve $\gamma_{k} \subset C^{\circ}$ connecting $b_{k}$ and $a_{k+1}$ with

$$
\operatorname{diam}\left(\gamma_{k}\right) \leq \operatorname{diam}\left(\mathcal{L}\left[b_{k}, a_{k+1}\right]\right) .
$$

Lastly, we let $\gamma$ be the curve obtained from $\tilde{\gamma}$ by replacing $\mathcal{L}\left[b_{k}, a_{k+1}\right]$ with $\gamma_{k}$ for all $k \geq 1$. Then $\gamma \subset C^{\circ}$.

To prove that $\gamma$ converges to $x$ we first observe that

$$
\left\|b_{k}-a_{k+1}\right\| \leq\left\|b_{k}-x_{k}\right\|+\left\|x_{k}-x_{k+1}\right\|+\left\|x_{k+1}-a_{k+1}\right\| \rightarrow 0 \quad \text { as } \quad k \rightarrow \infty .
$$

Thus from Lemma 5.4 and (5.10) we get

$$
\operatorname{diam}\left(\gamma_{k}\right) \leq \operatorname{diam}\left(\mathcal{L}\left[b_{k}, a_{k+1}\right]\right) \rightarrow 0 \quad \text { as } \quad k \rightarrow \infty .
$$

Now, let $\gamma_{x_{m}}$ denote the "tail" of $\gamma$ starting from $x_{m}$ and let $y \in \gamma_{x_{m}}$. It follows from definition that either $y \in \Omega\left(\mathcal{Q}_{n_{k}}\right)$ or $y \in \gamma_{k}$ for some $k$ with $n_{k} \geq m$. In the former case,

$$
\|x-y\| \leq\left\|x-x_{k}\right\|+\left\|x_{k}-y\right\| \leq\left\|x-x_{k}\right\|+2^{-\left(n_{k}-1\right) / 2} ;
$$

in the latter case,

$$
\|x-y\| \leq \operatorname{diam}\left(\gamma_{k}\right)+\left\|b_{k}-x_{k}\right\|+\left\|x-x_{k}\right\| \leq \operatorname{diam}\left(\gamma_{k}\right)+2^{-\left(n_{k}-1\right) / 2}+\left\|x-x_{k}\right\| .
$$

Consequently, $\sup \left\{\|x-y\|: y \in \gamma_{x_{m}}\right\} \rightarrow 0$ as $m \rightarrow \infty$. This proves that $x$ is simple.

To prove the second assertion, we first recall from Corollary 4.5 that $C^{\circ}$ is an open disk. Therefore there exists a conformal one-to-one Riemann mapping $h$ from $C^{\circ}$ onto the open unit disk $U:=\{x:\|x\|<1\}$. Since each $x \in \partial C$ is simple, $h$ can be extended to a homeomorphism from $C$ onto $\bar{U}$ (see Chapter 14 of [R]). This completes the proof.

Proof of Theorem 1.1. Theorem 1.4 follows immediately by combining Lemma 5.1 with Theorem 5.5. The theorem now follows by combining Theorems 1.3 and 1.4.

\section{Acknowledgments}

The authors are very grateful to Professor Robert Strichartz for many helpful suggestions on improving the original manuscript and simplifying some of the proofs. Part of this research was carried out while the first author was visiting the Southeast Applied Analysis Center and the School of Mathematics of Georgia Institute of Technology. He thanks the center, the school, the institute, and Professors Leonid Bunimovich and Yang Wang for their hospitality and support. Part of this work was also done while the authors were visiting The Chinese University of Hong Kong. They thank Professor Ka-Sing Lau and 
members of the Department of Mathematics for their hospitality. The second author is grateful to Professor David Finston for helping him to obtain Minigrant RC01-014 from New Mexico State University to visit The Chinese University of Hong Kong and do this research.

\section{References}

[BKS] S. Bailey, T. Kim, and R. S. Strichartz, Inside the Lévy dragon, Amer. Math. Monthly 109 (2002), 689-703.

[B] C. Bandt, Self-similar sets 5. Integer matrices and fractal tilings of $\mathbb{R}^{n}$, Proc. Amer. Math. Soc. 112 (1991), 549-562.

[BG] C. Bandt and G. Gelbrich, Classification of self-affine lattice tilings, J. London Math. Soc. (2) $\mathbf{5 0}$ (1994), 581-593.

[BW] C. Bandt and Y. Wang, Disk-like self-affine tiles in $\mathbb{R}^{2}$, Discrete Comput. Geom. 26(4) (2001), 591-601.

[BP] A. Benedek and R. Panzone, On some notable plane sets, II: Dragons, Rev. Un. Mat. Argentina 39 (1994), 76-89.

[DK] C. Davis and D. E. Knuth, Number representations and dragon curves-I, II, J. Recreational Math. 3 (1970), 66-81, 133-149.

[E] G. A. Edgar, Measure, Topology, and Fractal Geometry, Springer-Verlag, New York, 1990.

[F] K. J. Falconer, Fractal Geometry. Mathematical Foundations and Applications, Wiley, Chichester, 1990.

[G] M. Gardner, Mathematical games, Scientific American 216(3) (1967), 124-125; 216(4) (1967), 118-120; 217(1) (1967), 115.

[Ge1] G. Gelbrich, Crystallographic reptiles, Geom. Dedicata 51 (1994), 235-256.

[Ge2] G. Gelbrich, Self-affine lattice reptiles with two pieces in $\mathbb{R}^{n}$, Math. Nachr. 178 (1996), 129-134.

[H] J. E. Hutchinson, Fractals and self-similarity, Indiana Univ. Math. J. 30 (1981), 713-747.

[KL] I. Kirat and K.-S. Lau, On the connectedness of self-affine tiles, J. London Math. Soc. (2) 62 (2000), 291-304.

[LRT] J. Luo, H. Rao, and B. Tan, Topological structure of self-similar sets, Fractals 10(2) (2002), 223-227.

[M] B. B. Mandelbrot, The Fractal Geometry of Nature, Freeman, San Francisco, CA, 1982.

[MW] R. D. Mauldin and S. C. Williams, Hausdorff dimension in graph directed constructions, Trans. Amer. Math. Soc. 309 (1988), 811-829.

[NSVW] S.-M. Ngai, V. Sirvent, J. J. P. Veerman, and Y. Wang, On 2-reptiles in the plane, Geom. Dedicata 82 (2000), 325-344.

[R] W. Rudin, Real and Complex Analysis, third edition, McGraw-Hill, New York, 1987.

[S] R. S. Strichartz, Self-similar measures and their Fourier transforms III, Indiana Univ. Math. J. 42 (1993), 367-411.

[SW] R. S. Strichartz and Y. Wang., Geometry of self-affine tiles I, Indiana Univ. Math. J. 48 (1999), $1-23$.

Received March 11, 2002, and in revised form October 30, 2002. Online publication March 19, 2003. 